\title{
Adubação química, adubação orgânica e extrato pirolenhoso no desenvolvimento de mudas de palmeiras fênix (Phoenix roebelenii)
}

\section{Chemical fertilization, organic fertilization and pirolygneous extract on vegetative development palm phoenix (Phoenix roebelenii)}

\author{
Christina da Silva Wanderley ${ }^{1 *}$; Ricardo Tadeu de Faria ${ }^{2}$; Maurício Ursi Ventura ${ }^{2}$
}

\begin{abstract}
Resumo
O efeito da utilização da adubação química, da adubação orgânica e do extrato pirolenhoso (EPL) foi avaliado no comprimento e número de folhas de mudas de palmeiras fênix. As plantas receberam adubação orgânica com torta de mamona, química com o fertilizante mineral NPK na fórmula 20-0520 e EPL nas concentrações de 0,1 e $0,2 \%$. O comprimento da maior folha e o número de folhas foram avaliados mensalmente durante um ano desde o início do experimento. Os dados foram submetidos à análise de variância e ao teste Tukey, a 5\% de significância. O tratamento adubação orgânica isolada e adubação orgânica +EPL promoveu aumento no comprimento e no número de folhas das plantas de palmeiras fênix. O fertilizante mineral NPK, combinado ou não com o EPL, não proporcionou incrementos no comprimento e número de folhas das plantas. O EPL isoladamente não alterou os parâmetros avaliados.
\end{abstract}

Palavras-chave: Plantas ornamentais, altura de plantas, número de folhas

\begin{abstract}
The effect of the use of chemical fertilization, organic fertilization and pirolygneous extract (EPL) was evaluated in the width and number of leaves of seedlings of phoenix palms. The plants were fertilizer with organic castor bean, chemical fertilizer NPK 20-05-20 formula and EPL concentrations of 0,1 and $0,2 \%$. The length of the longest leaf and leaf number were evaluated monyhly for one from the start of the experiment. The data were analyzed by ANOVA and Tukey test at $5 \%$ significance level. Treatment organic fertilizer and EPL $0,1 \%$ + organic fertilization increased the number of leaves of palm phoenix plants. Mineral fertilizer NPK combined or not with EPL provided no increases in length and number of leaves. The EPL itself did not change the parameters.
\end{abstract}

Key words: Ornamental plants, plant height, number of leaves

1 Doutoranda de Agronomia da Universidade Estadual de Londrina, UEL. Londrina, PR. E-mail: chriscsw@sercomtel.com.br

2 Profs. Drs. do Dept ${ }^{\circ}$ de Agronomia, UEL, Londrina, PR. E-mail: faria@uel.br; mventura@uel.br

* Autor para correspondência 
A palmeira fênix (Phoenix roebelenii) é uma planta solitária, delicada, com altura entre dois e quatro metros, cujo caule é recoberto na juventude por um tecido fibroso espesso e pela base das folhas já caídas e quando idoso, pelas cicatrizes remanescentes (LORENZI et al., 2004). Essa palmeira deve ser plantada em substrato com boa drenagem e aeração, pois apresenta crescimento lento, por vezes atingindo o padrão de venda somente aos dois ou três anos e por isso pode crescer dentro de vasos (MEEROW; BROSCHAT, 1996). É uma planta de alto interesse ornamental, visto que é tolerante tanto ao sol direto como à meia sombra.

Diversos tipos de adubos podem ser usados no cultivo desse tipo plantas, e embora haja falta de informação a respeito das exigências nutricionais da espécie em questão, muitos autores têm dado ênfase à utilização de adubos orgânicos em palmeiras desde o plantio, como o esterco de curral curtido (OLIVEIRA; FARIAS NETO, 2004). Entretanto, quando se cultivam plantas em vasos, devem-se procurar adubos como a torta de mamona e o húmus de minhoca que oferecem menor cheiro, e os adubos químicos, que não oferecem cheiro.

Quando se refere à adubação química, há diversas recomendações nas bibliografias de diferentes fórmulas de NPK para palmeiras, mas não são encontradas com facilidade no comércio, sendo as mais comuns 10-10-10; 04-14-08; 20-0520 e 08-28-16.

Há ainda outros produtos que têm sido utilizados na agricultura, e que tem se destacado por serem subprodutos obtidos da produção de carvão vegetal, como o fino de carvão e o extrato pirolenhoso (EPL) (ZANETTI et al., 2003). O extrato pirolenhoso é o líquido obtido através da condensação da fumaça durante o processo de carbonização da madeira para produção de carvão. Este produto diluído em água e aplicado ao solo, melhora suas propriedades físicas, químicas e biológicas, proporciona aumento da população de microrganismos benéficos e favorece a absorção de nutrientes do solo pelo sistema radicular das plantas (MIYASAKA et al., 2001). Outros estudos mostram os efeitos benéficos do extrato pirolenhoso associado ao fino de carvão aplicado ao solo também como fertilizante orgânico em arroz (TSUZUKI et al., 2000; ICHIKAWA; OTA, 1982), cana-de-açúcar (UDDIN et al., 1995), batata doce (SHIBAYAMA, 1998); melão (DU et al., 1998; TSUZUKI et al., 1993) e sorgo (ESECHIE et al., 1998). No Brasil, entretanto, há poucos estudos sobre as utilizações do extrato pirolenhoso com plantas ornamentais (SCHNITZER et al., 2010), e nenhum relato com palmeira fênix.

O objetivo desse trabalho foi avaliar o efeito da adubação química, da adubação orgânica e do extrato pirolenhoso no comprimento e número de folhas de mudas de palmeira fênix.

O trabalho foi desenvolvido na área experimental do Departamento de Agronomia da Universidade Estadual de Londrina $\left(23^{\circ} 23^{\prime} \mathrm{S}, 51^{\circ} 11^{\prime} \mathrm{O}\right.$ e altitude média de 566m), no período de outubro de 2007 a setembro de 2008.

O cultivo das plantas foi realizado em vasos plásticos, com capacidade de 8 litros, que foram preenchidos com uma mistura de terra de barranco, casca de arroz carbonizada e casca de pinus triturada, em proporções iguais. Nesses vasos foram plantadas mudas de palmeira fênix com uma haste, comprimento médio de folhas de $46,7 \mathrm{~cm}$ e 7 folhas por planta; mantidas a céu aberto e submetidas a irrigações por aspersão, automatizadas, 4 vezes ao dia, por 10 minutos, totalizando em torno de 1 litro de água por dia por vaso.

O delineamento experimental utilizado foi em blocos casualizados, com 10 repetições por tratamento. Os tratamentos foram: $\mathrm{T} 1=$ sem adubação (testemunha); T2= EPL $0,1 \% \quad(1 \mathrm{ml}$ diluído em 1 litro de água); T3= EPL 0,2\% ( $2 \mathrm{ml}$ diluídos em 1 litro de água); T4= NPK 20-05-20; T5 $=$ NPK 20-05-20 + EPL 0,1\%; T6= NPK 20-05$20+$ EPL $0,2 \% ; \mathrm{T} 7=$ torta de mamona; $\mathrm{T} 8=$ torta de mamona + EPL $0,1 \%$ e T9= torta de mamona + EPL 0,2\%. 
O Extrato Pirolenhoso (EPL) foi obtido junto à Carvoaria Coroados (Londrina-PR), que utiliza madeira de reflorestamento de Eucalyptus grandis W.Hill ex Maiden, cuja composição química básica é nitrogênio $3,5 \%$; potássio $0,003 \%$; cobre $0,09 \%$; zinco $0,10 \%$; cálcio 0,16 ; magnésio $0,40 \%$ e enxofre $10,10 \%$. As aplicações de EPL foram realizadas quinzenalmente, quando o EPL era diluído em água e cada vaso recebia $100 \mathrm{~mL}$, através de rega. As empresas fabricantes do extrato, como a Agropirol K-5 e a Biopirol recomendam que sua aplicação no substrato de plantas ornamentais seja feita quinzenalmente, nas doses de $100 \mathrm{~mL} / 100 \mathrm{~L}$ à 300 mL/100 L.A adubação orgânica (torta de mamona), que é obtida a partir de resíduos da prensagem da baga da mamona, cuja composição química básica fornecida na embalagem do produto é nitrogênio $5 \%$; carbono $35 \%, \mathrm{pH}$, foi feita mensalmente (40 $\mathrm{g}$ vaso $^{-1}$ ), visto que a empresa produtora de torta de mamona (Vitaplan), recomenda sua aplicação em vasos médios (com diâmetro até $25 \mathrm{~cm}$ ), para plantas ornamentais, uma vez ao mês, na quantidade de 3 colheres de sopa (40 g). A adubação química (NPK 20-05-20) foi feita trimestralmente (40 g vaso $\left.^{-1}\right)$. A duração do experimento foi de um ano. Foram feitas avaliações mensais do comprimento da maior folha e o número de folhas. Os dados foram submetidos à análise de variância e ao teste Tukey, a 5\% de significância.

$\mathrm{Na}$ (Figura 1) estão apresentados os valores obtidos para os comprimentos das folhas um ano após o início do experimento.

Figura 1. Valores obtidos durante o experimento para o comprimento da maior folha de palmeira fênix. Legenda: $\mathrm{T} 1=$ sem adubação (testemunha); T2= EPL0,1\%; T3= EPL0,2\%; T4= NPK-20-05-20; T5= NPK-20-05-20 + EPL0,1\%; $\mathrm{T} 6=\mathrm{NPK}-20-05-20+\mathrm{EPL} 0,2 \% ; \mathrm{T} 7=$ torta de mamona; $\mathrm{T} 8=$ torta de mamona + EPL0,1\% e T9= torta de mamona + EPL0,2\%.

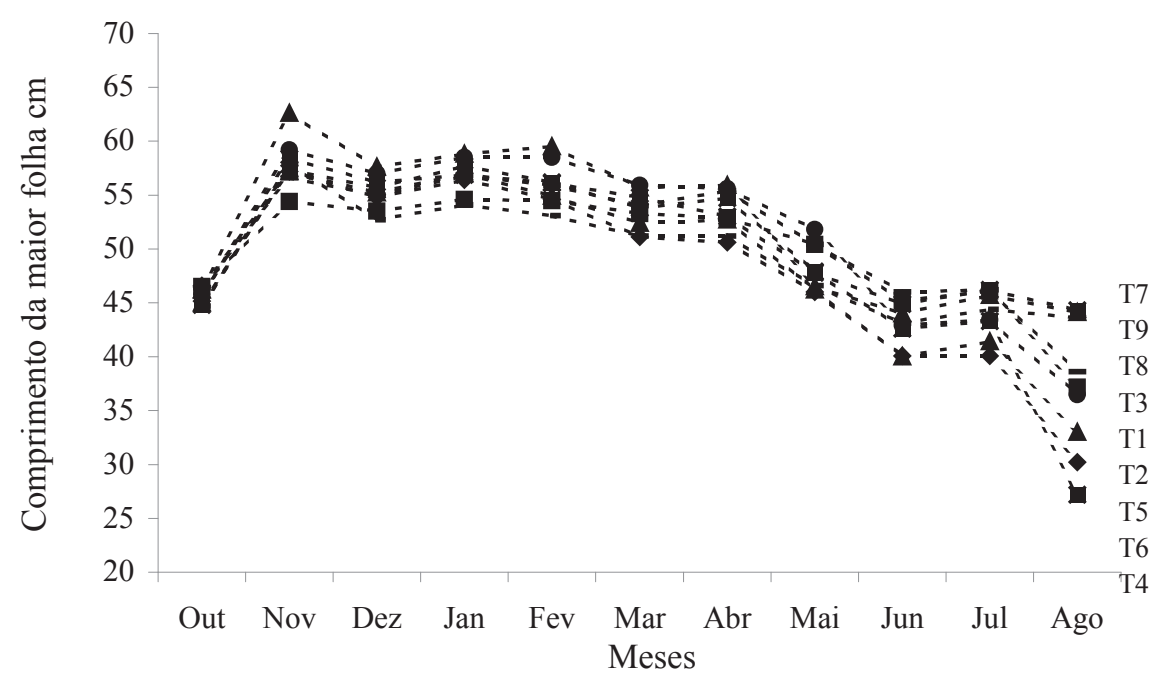

Fonte: Elaboração dos autores. 
Quando se comparou o tratamento T1 (testemunha) com os tratamentos que receberam o EPL a 0,1 e a $0,2 \%$ (T2 e T3), verificou-se que entre os três tratamentos poucas diferenças foram encontradas, mas o que foi verificado foram as reduções no comprimento da maior folha no decorrer do experimento, sendo que do início ao final do experimento aconteceram reduções de mais de $7 \mathrm{~cm}$ em todas as folhas emitidas ( no T1 redução de 9,3 cm; no T2 redução de 9,2 cm e no T3 redução de $7,8 \mathrm{~cm})$.

Nos tratamentos com adubação química NPK 20-05-20 com a adição ou não de EPL (T4 NPK, T5 NPK+EPL0,1\% e T6 NPK+EPL0,2\%) constatouse não haver diferenças entre o comprimento da maior folha das plantas para aquelas que receberam somente adubação química e as que receberam além da adubação química, a aplicação de EPL. Pode-se observar que houve um grande decréscimo no parâmetro comprimento da folha maior durante o experimento, pois as plantas chegaram ao final desse período com folhas mais de $10 \mathrm{~cm}$ menores do que quando se iniciaram as observações (no T4 redução de 17,6cm; no T5 redução de $12 \mathrm{~cm}$ e no T6 redução de $16,4 \mathrm{~cm}$ ), sendo que nas plantas testemunha essa redução foi de 9,3 cm em média. Esse resultado pode indicar que a adubação química foi excessiva, causando toxidez às plantas.

Apesar de os adubos químicos de formula NPK, no caso 20-05-20, possuírem alta porcentagem de nitrogênio, autores como Meerow e Broschat (1996) destacam que esse tipo de adubação não deva ser utilizado para palmeiras em vasos, pois devido a alta solubilidade desses adubos, a grande quantidade de sal solúvel causa injúrias às raízes, reduzindo o crescimento das plantas.

$\mathrm{Na}$ literatura não há referências sobre a quantidade e épocas para adubação química de palmeiras fênix, por isso as adubações para esse experimento foram baseadas nas recomendações feitas para outras espécies de plantas.

Entre os tratamentos com adubação orgânica
(AO) com torta de mamona com a adição ou não de EPL(T7AO, T8 AO+EPL0,1\% e T9AO+EPL0,2\%), pode-se observar que os três tratamentos seguem a mesma tendência de redução da comprimento da folha maior durante o tempo do experimento, sendo que para esses três tratamentos as reduções foram menores do que para os tratamentos apresentados anteriormente (no T7 redução de $1,5 \mathrm{~cm}$; no T8 redução de $1,1 \mathrm{~cm}$ e no T9 redução de $2,1 \mathrm{~cm}$ ), reduções bem menores inclusive do que nas testemunhas ( $9,3 \mathrm{~cm}$ de redução). Pela figura 1, notase que as plantas desses três tratamentos chegaram ao final do experimento com praticamente o mesmo comprimento de maior folha, sendo superior a todos os demais tratamentos e a testemunha. Esse desempenho melhor de plantas tratadas com torta de mamona com e sem EPL, pode ser explicado pelo fato de a torta de mamona possuir um alto teor de N (5\%), além de carbono orgânico (35\%), que favorecem o crescimento das plantas.

A (Figura 1) mostra que não houve diferenças entre os tratamentos Testemunha (T1) e os tratamentos com EPL (T2 e T3) e com adubo químico combinado com o EPL 0,1\% (T5). Entretanto, houve diferenças comparando com os três tratamentos que utilizaram o adubo orgânico torta de mamona (T7, T8 e T9), onde se observou diferenças significativas no comprimento da maior folha. Os tratamentos que utilizaram somente adubo químico isolado e combinado com EPL 0,2\% (T4 e T6), apresentaram comprimento de folhas menores do que as das plantas testemunhas, evidenciando uma possível toxidez às plantas, induzindo um menor crescimento das folhas novas.

A (Figura 2) apresenta os valores obtidos para o parâmetro número de folhas durante o experimento.

Quando se comparou o tratamento T1 (testemunha) com os tratamentos que receberam o EPL a 0,1 e a $0,2 \%$ (T2 e T3), verificou-se que não houve diferenças entre os tratamentos, sendo que a evolução de todos se deu de modo bastante homogêneo durante todo o experimento, com um pequeno destaque para o $\mathrm{T} 3$. 
Nos tratamentos com adubação química NPK 20-05-20 com a adição ou não de EPL (T4 NPK, T5 NPK+EPL0,1\% e T6 NPK+EPL0,2\%) pode-se observar que não houve diferenças entre o número de folhas para as plantas que receberam somente adubação química e as que receberam além da adubação química, a aplicação de EPL, durante todo o experimento. Observou-se aumento no número de folhas do início do experimento até o $6^{\circ}$.mês, com posterior decréscimo no número de folhas até o final do experimento. Essas plantas, ao final do experimento tinham menos folhas emitidas do que as plantas testemunha e uma quantidade muito menor do que as plantas que receberam a adubação orgânica com torta de mamona. Novamente se pode inferir que a adubação química causou toxidez às plantas, inibindo a emissão de folhas novas.

Figura 2. Valores obtidos durante o experimento para o número de folhas de palmeira fênix. Legenda: $\mathrm{T} 1=$ sem adubação (testemunha); T2= EPL0,1\%; T3= EPL0,2\%; T4= NPK-20-05-20; T5= NPK-20-05-20 + EPL0,1\%; T6= NPK-20-05-20 + EPL0,2\%; T7= torta de mamona; T8= torta de mamona + EPL0,1\% e T9= torta de mamona + EPL0,2\%.

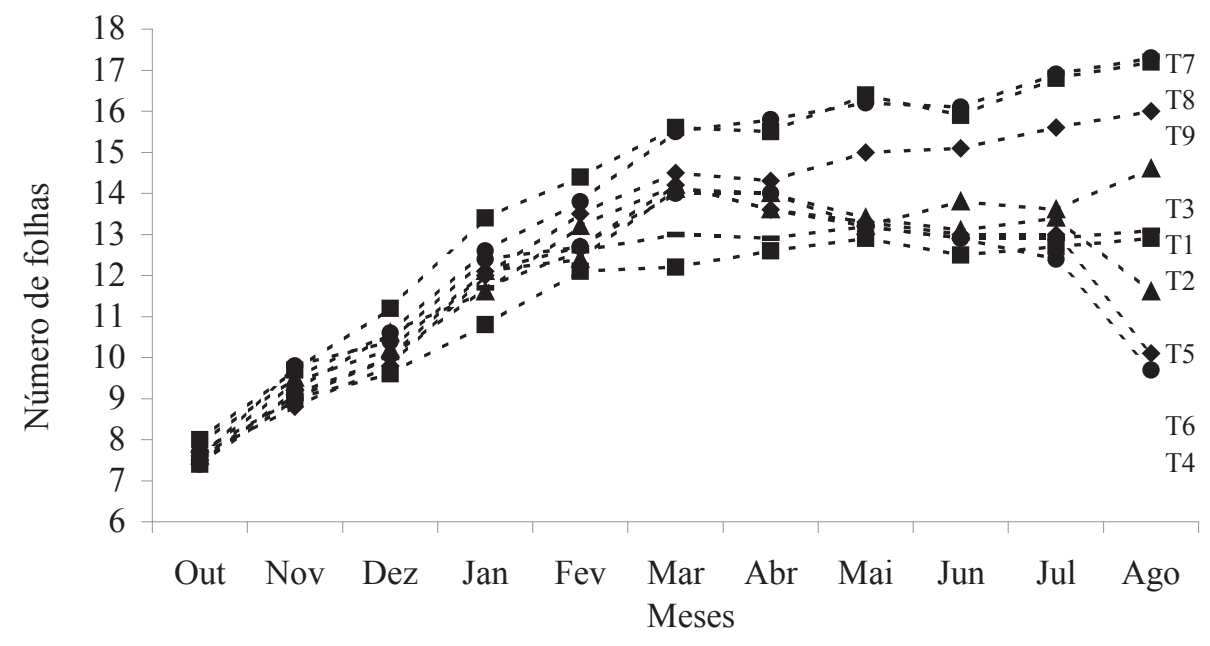

Fonte: Elaboração dos autores.

Nos tratamentos com adubação orgânica torta de mamona com a adição ou não de EPL (T7 AO, T8 AO+EPL 0,1\% e T9 AO+EPL 0,2\%), verificou-se que os três tratamentos seguiram a mesma tendência de aumento do número de folhas durante o tempo do experimento, sendo que ao final do período avaliado, o T7 e o T8 apresentaram valores maiores para o parâmetro em questão, com mais do que o dobro do número de folhas verificados no início do experimento, e muito maior do que os verificados em todos os outros tratamentos.
A (Figura 2) mostra que o tratamento testemunha $\mathrm{T} 1$, que ao final do experimento apresentava 13,1 folhas, não diferiu do T2 (com 12,9 folhas) e do T3 (com 14,6 folhas). Entretanto, diferiu dos demais tratamentos, com destaque para o T4 (9,7 folhas), T5 (11,2 folhas) e T6 (10,1 folhas) onde foi utilizada a adubação química e que apresentaram menos folhas do que a testemunha ao final do experimento. Da mesma forma, houve uma diferença maior para o T7 (17,3 folhas) e T8 (17,2 folhas) que utilizaram adubação orgânica com e sem aplicação de EPL e 
apresentaram número de folhas superior aos demais tratamentos. Pelo número de folhas encontradas, pode-se inferir que a espécie Phoenix roebelenii apresenta a emissão de uma folha a cada 2 meses em condições normais (T1), sendo que esse número foi mais do que o dobro nos tratamentos $\mathrm{T} 7$ e T8 ao final do experimento. Novamente a adubação química aparentemente causou toxidez às plantas, e durante um ano, as plantas que receberam esse tratamento emitiram pouquíssimas folhas, já que o experimento começou com todas as plantas com média de 7,6 folhas.

Outros resultados com a utilização do EPL foram encontrados para diversas culturas para diferentes características.

Ichikawa e Ota (1982) obtiveram maior enraizamento em plantas de arroz quando utilizaram o EPL aplicado isoladamente no substrato de crescimento. Uddin et al. (1995) verificaram aumento no conteúdo de açúcar em plantas de canade-açúcar tratadas com EPL. Tsuzuki et al.(2000), estudando a cultura do arroz, obtiveram maior aumento na quantidade e no comprimento de raízes quando tratadas com EPL mais adubação química.

Em relação a plantas ornamentais, Kadotta e Niimi (2004) verificaram maior número de botões florais em Melampodium suffruticosa (Baker) Stuessi, Salvia coccinea Buc'hoz ex Etl. e Zinnia peruviana (Lineus) L. quando tratadas com EPL. Esses mesmos autores, utilizando uma mistura de carvão com esterco de curral (adubo orgânico) observaram uma boa qualidade em flores como Tagetes patula, Zinnia lineareis e Melampodium paludosu. Observaram também que a produção de Tagetes registrou aumentos, bem como aumento no número de ramos em Zinnia.

Prado et al. (2009), em experimento com uma planta conífera, utilizando adubação química com adubo formulado mais uréia em vasos, verificaram um maior crescimento das plantas, o que não foi encontrado no presente trabalho.
Em experimentos com batata doce, Du et al. (1998) verificaram que a mistura de EPL com adubação orgânica proporcionou aumento de peso seco tanto da parte aérea como de tubérculos. Da mesma forma Mascarenhas et al. (2006) obtiveram maior produção total e maior quantidade de frutos de primeira em plantas de quiabeiro quando tratadas com a mistura de EPL mais adubação orgânica.

A adubação orgânica (torta de mamona) combinada ou não com o EPL mostrou-se eficiente no aumento do comprimento da maior folha e na emissão de folhas da palmeira fênix.

O extrato pirolenhoso (EPL), combinado ou não com a adubação química (NPK) não demonstrou efeitos sobre o comprimento e número de folhas da palmeira fênix.

O EPL isoladamente não alterou os parâmetros avaliados.

A adubação orgânica com torta de mamona apresentou resultado superior a adubação química NPK no desenvolvimento das mudas de palmeira fênix.

\section{Agradecimentos}

Agradecemos ao colega engenheiro agrônomo Paulo Hisashi Tashina pelo grande auxílio nas traduções dos artigos científicos em japonês.

\section{Referências}

DU, H. G.; MORI, E.; TERAO, H.; TSUZUKI, E. Effect of the mixture of charcoal with pyroligneous acido on shoot and root growth of sweet potato. Japan Journal of Crop Science, Bankyo-ku, Tokyo, v. 67, n. 2, p. 149-152, 1998.

ESECHIE, H. A.; DHALIWAL, G. S.; ARORA, R.; RANDHAWA, N. S.; DHAWAN, A. K. Assessment of pyroligneous liquid as a potential organic fertilizer. In: ECOLOGICAL AGRICULTURE AND SUSTAINABLE DEVELOPMENT, 1997, Chandigarh, India. Proceedings... Chandigarh: Center for Research in Rural and Industrial Development, 1998. v. 1, p. 591-595. 
ICHIKAWA, T.; OTA, Y. Plant growth-regulating activity of pyroligneous acid. I- Effect of pyroligneous acid on the growth of rice seedlings. Japan Journal of Crop Science, Bankyo-ku, Tokyo, v. 51, n. 1, p. 14-17, 1982.

KADOTTA, M.; NIIMI, Y. Effects of charcoal with pyroligneous acid and barnyard manure on bedding plants. Scientia Horticulturae, Japan, v. 101, p. 327- 332, 2004.

LORENZI, H.; SOUZA, H. M. de; COSTA, J. T. de M.; CERQUEIRA, L. S. C. de; FERREIRA, E. Palmeiras brasileiras e exóticas cultivadas. Nova Odessa: Instituto Plantarum, 2004, 416 p.

MASCARENHAS, M. H. T.; LARA, J. F. R.; PURCINO, H. M. A.; SIMÕES, J. C.; MOREIRA, D. C.; FACION, C. E. Efeito da utilização do extrato pirolenhoso na produtividade do quiabeiro. Revista Brasileira de Horticultura, Cruz das Almas, v. 24, n. 1, p. 3126-3128, 2006.

MEEROW, A. W.; BROSCHAT, T. K. Container production of palms. Flroida, USA: University of Florida, Extension, Institute of food anal agricultural sciences, CIR 1163, 1996.

MIYASAKA, S.; OHKAWARA, T.; NAGA, K.; YAZAKI, H.; SAKITA, M. N. Técnicas de produção e uso do fino de carvão e licor pirolenhoso. In: ENCONTRO DE PROCESSOS DE PROTEÇÃO DE PLANTAS. 1., 2001. Botucatu. Resumos... Botucatu: [s.n], 2001. v. 1, p. 16-176.

OLIVEIRA, M. J. do S. P.; FARIAS NETO, J. T. de. Cultivar BRS-Pará: açaizeiro para produção de frutos em terra firme. Belém: EMBRAPA, 2004. (Comunicado técnico, 114).
PRADO, A. E. do; PEREIRA, G. P.; CARVALHO, D. B. de; CARVALHO, R. I. N. de. Crescimento de mudas de Chamaecyparis lawsoniana em diferentes níveis de adubação mineral do substrato e em cobertura. Semina: Ciências Agrárias, Londrina, v. 30, p. 1163-1172, 2009. Suplemento 1.

SCHNITZER, J.; FARIA, R. T. de; VENTURA, M. U.; SORACE, M. Substratos e extrato pirolenhoso no cultivo de orquídeas brasileiras Cattleya intermédia (John Lindley) e Miltonia clowesii (John Lindley) (Orchidaceae). Acta Scientiarum, Maringá, v. 32, n. 1, p. 139-143, 2010.

SHIBAYAMA, H. Effects of application of pyroligneus acid solution produced in Karatsu city and growth and free sugar contents of storage roots of sweet potatoes. Marine and Higland Bioscience Center Report, Phukel, v. 7, p. 15-23, 1998.

TSUZUKI, E.; WAKIYAMA, Y.; ETO, H.; HARADA, H. Effect of organic matters on growth and quality of crops: II effect of charcoal with pyroligneus acid on quality of melon (Cucumis melo L.) Japan Journal of Crop Science, Bankyo-ku, Tokyo, v. 62, p. 170-171, 1993.

Effect of chemical compounds in pyroligneous acido on root growth in rice plants. Japan Journal of Crop Science, Bankyo-ku, Tokyo, v. 66, n. 4, p. 15-16, 2000.

UDDIN, S. M. M.; MURAYAMA, S.; ISHIMINE, Y.; TSUZUKI, E.; HARADA, J. Studies on sugarcane cultivation. II- Effects of the mixture of charcoal with pyroligneous acid on dry matter production and root growth of summer planted sugarcane (Saccharum officinarum L.). Japan Journal of Crop Science, Bankyoku, Tokyo, v. 64, n. 4, p. 747-753, 1995.

ZANETTI, M.; CAZETTA, J. O.; MATOS JUNIOR, D.; CARVALHO, S. A. Uso de subprodutos de carvão vegetal na formação do porta enxerto limoeiro "Cravo" em ambiente protegido. Revista Brasileira de Fruticultura, Jaboticabal, v. 25, n. 3, p. 508-512, 2003. 
